\title{
Evaluation of Recombinant Mycobacterium tuberculosis Antigens MPT64, CFP10, and ESAT6 for Delayed-Type Hypersensitivity Responses in Guinea Pigs
}

\author{
Wandee Yindeeyoungyeon ${ }^{*}$, Kamolchanok Rukseree ${ }^{2}$, Siriporn Tungsudjai ${ }^{3}$, \\ Tasanee Panichakul ${ }^{4}$ \\ ${ }^{1}$ National Center for Genetic Engineering and Biotechnology (BIOTEC), National Science and Technology \\ Development Agency (NSTDA), Pathum Thani, Thailand \\ ${ }^{2}$ Mahidol University, Amnatcharoen Campus, Amnatcharoen, Thailand \\ ${ }^{3}$ Faculty of Veterinary Science, Mahidol University, Bangkok, Thailand \\ ${ }^{4}$ Faculty of Science and Technology, Suan Dusit University, Bangkok, Thailand \\ Email: wandee@biotec.or.th
}

Received 29 June 2015; accepted 4 August 2015; published 7 August 2015

Copyright (C) 2015 by authors and Scientific Research Publishing Inc.

This work is licensed under the Creative Commons Attribution International License (CC BY).

http://creativecommons.org/licenses/by/4.0/

(c) (i) Open Access

\section{Abstract}

The tuberculin Purified Protein Derivative (PPD) skin test is widely used; however, the results are often inaccurate. Positive results can be observed in patients with active tuberculosis (TB) as well as in BCG-vaccinated persons and individuals who are infected with mycobacteria but have not developed the disease. MPT64, an antigen secreted from actively growing Mycobacterium tuberculosis and some strains of $M$. bovis BCG such as BCG Tokyo and BCG Russia, is immunogenic and elicits delayed-type hypersensitivity (DTH) in guinea pigs and humans. This antigen has been used to develop a new skin test for the diagnosis of active TB infection. Two of the antigens encoded by the $M$. tuberculosis-specific region of difference 1 (RD1, deleted in M. bovis BCG strains), CFP10 (culture filtrate protein 10) and ESAT6 (early secreted antigenic target-6), also induce $M$. tuberculosis-specific DTH responses. The aim of this study was to investigate the DTH responses in guinea pigs infected with $M$. tuberculosis or $M$. bovis BCG Tokyo elicited by three purified recombinant proteins (rMPT64, rCFP10 and rESAT6) compared to those elicited by PPD. In this study genes encoding MPT64, CFP10, and ESAT6 were cloned and expressed as recombinant proteins with the addition of a C-terminal $\mathrm{His}_{6}$ tag for ease of purification by Immobilized Metal ion Affinity Chromatography (IMAC). The recombinant proteins (rMPT64, rCFP10, and rESAT6) were purified to homogeneity and were used to elicit DTH responses in guinea pigs infected with $M$. tuberculosis or

\footnotetext{
*Corresponding author.

How to cite this paper: Yindeeyoungyeon, W., Rukseree, K., Tungsudjai, S. and Panichakul, T. (2015) Evaluation of Recombinant Mycobacterium tuberculosis Antigens MPT64, CFP10, and ESAT6 for Delayed-Type Hypersensitivity Responses in Guinea Pigs. Advances in Microbiology, 5, 586-598. http://dx.doi.org/10.4236/aim.2015.58061
} 
M. bovis BCG Tokyo. The results showed that rMPT64 elicits a DTH response comparable to that of PPD in M. bovis BCG Tokyo-vaccinated animals. However, M. tuberculosis-infected animals show less reactivity to rMPT64 than they do to PPD. Although single rCFP10 or rESAT6 did not readily elicit a DTH response in $M$. tuberculosis-infected animals, combining these antigens with rMPT64 led to an increased DTH response, thus enabling the detection of TB infection.

\section{Keywords}

\section{M. tuberculosis, M. bovis BCG Tokyo, Delayed-Type Hypersensitivity, DTH, MPT64, CFP10, ESAT6}

\section{Introduction}

Tuberculin skin tests by intradermal Purified Protein Derivative (PPD) injection can rapidly detect mycobacterial infection, but it gives a positive result, not only to active TB patients, but also to persons with asymptomatic Mycobacterium tuberculosis infection and M. bovis BCG-vaccinated persons. PPD is composed of $>100$ proteins and represents all proteins secreted during growth in culture media in vitro, as PPD is prepared through the precipitation of culture filtrates from M. tuberculosis. Different culture batches and precipitation methods result in varied levels of tuberculoprotein (48\% to 99\%) [1]. Thus, the identification of specific defined antigens that can discriminate between active TB-infected patients and latent or asymptomatic TB-infected/vaccinated persons will lead to more efficient control and better treatment of the disease.

In recent years, several antigens secreted from actively growing $M$. tuberculosis cultures have been characterized. To achieve a high sensitivity of diagnosis for TB patients, several antigens have been evaluated for their ability to elicit humoral and cell-mediated immune responses. Antigens such as MPT51, 81/88 kDa GlcB protein, and $38 \mathrm{kDa}$ protein elicit antibody responses, whereas the antigens of Ag85, ESAT6, CFP10, and MPT64 elicit T-cell responses [2]. In the search for a new skin test that is specific for the diagnosis of TB infection, ESAT6, MPT64, CFP10, and DPPD (Rv0061) elicit delayed-type hypersensitivity (DTH) in a guinea pig model [2] [3].

Recent studies reported that $M$. tuberculosis-specific antigens ESAT6, CFP10, CFP21, and MPT64, alone or in combination, induce a strong DTH response in M. tuberculosis-infected guinea pigs but elicit little response in BCG-vaccinated animals [4] [5]. This observation emphasizes the specificity of these antigens as viable candidates for skin test development. In fact, a TB skin patch test using several TB-specific antigens (including MPT64) has been developed, and a patent has been filed (United State Patent 6979450); however, this test has not been widely used (in Thailand, PPD is still used). Thus, development of an improved skin patch test for TB diagnosis that is suitable for local use is worthwhile.

Calmette and Guerin of the Pasteur Institute passaged a strain of M. bovis 230 times in vitro from 1908 to 1921, thus developing the vaccine strain, M. bovis BCG. Since then, the M. bovis BCG seed lots were distributed to institutions around the world. Serial passage was performed to maintain the BCG strain at different vaccine facilities until lyophilization was introduced in the 1960s. Molecular analysis of genomic DNA by whole-genome DNA microarray revealed that several segments of DNA were missing from M. bovis and M. bovis BCG strains compared to $M$. tuberculosis H37Rv reference strain. These deletion regions were named RD1 to RD16. Nine regions (RD4, RD5, RD6, RD7, RD9, RD10, RD11, RD12, and RD15) encompassing 61 open reading frames (ORFs) are absent from all M. bovis BCG and M. bovis strains, whereas two regions (RD9 and RD13) with 30 ORFs are absent from $M$. bovis BCG and some $M$. bovis strains. Five regions (RD1, RD2, RD8, RD14, and RD16) containing 38 ORFs are present in M. bovis but absent from some or all M. bovis BCG strains. RD1, missing from all M. bovis BCG strains, contains 9 ORFs which include two secretory proteins Rv3874 (CFP10) and Rv3875 (ESAT6). RD2, present in "early strains” such as BCG Russia and BCG Tokyo, contains 11 ORFs which include Rv1980c (MPT64) [6]. Since all M. bovis BCG strains do not secrete CFP10 or ESAT6, these two proteins are appropriate candidates for differentiating between infection with $M$. tuberculosis and vaccination with $M$. bovis BCG.

In Thailand, the M. bovis BCG strain used is Tokyo; therefore, in this study, we used the Tokyo strain and compared it to $M$. tuberculosis H37Rv (laboratory virulent strain). It was previously shown that recombinant MPT64 (rMPT64) was able to elicit a DTH response in guinea pigs sensitized with M. bovis BCG Tokyo similar 
to that of native MPB64 and native MPT64 isolated from culture filtrates of M. bovis BCG Tokyo (which expresses MPB64) and M. tuberculosis H37Rv (which expresses MPT64), respectively [7] [8]. Thus, in this study, we tested the responses of guinea pigs infected with M. tuberculosis or M. bovis BCG Tokyo by sensitization with PPD or purified recombinant MPT64, CFP10, and ESAT6. The aim of this study was to test whether purified recombinant antigens can induce a DTH reaction comparable to PPD in guinea pigs. This result could lead to the use of specific, purified recombinant antigens to develop an improved skin test for TB diagnosis that could replace the currently used PPD.

\section{Materials and Methods}

\subsection{Molecular Biology Materials and Chemicals}

Restriction enzymes, DNA molecular weight markers, and protein molecular weight markers were purchased from New England Biolabs, USA. Takara LA Taq and T4 DNA ligase were obtained from Takara Bio Inc., Japan. The plasmid purification kit was purchased from QIAGEN, USA. Other chemicals and antibiotics were obtained from Sigma-Aldrich Inc., USA. PPD (tuberculin skin test reagent lot\# TP0081108/12/201108/06/2012) was acquired from Queen Saovabha Memorial Institute, the Thai Red Cross Society. Protein concentrator MWCO 5000 Da was obtained from Sartorius Stedim Biotech GmbH, Germany (Vivaspin) or Millipore, USA (Amicon ultrafiltration). Talon Superflow Metal Affinity resin was purchased from Clontech Takara Bio Inc., Japan. The HiLoad 16/60 Superdex 75 prep grade gel filtration column was obtained from GE Healthcare, Sweden. The two commercial plasmids used in this study were pGEM-T Easy (PCR cloning vector, Amp ${ }^{\mathrm{R}} / \mathrm{Ampici}$ lin resistant, Promega) and pET24b (C-terminal $\mathrm{His}_{6}$-tagged expression vector, $\mathrm{Km}^{\mathrm{R}} /$ Kanamycin resistant, Novagen).

\subsection{Bacterial Strains, Culture Media, and Guinea Pigs}

Mycobacterium tuberculosis strain H37Rv (laboratory virulent strain, ATCC 25618) and M. bovis BCG Tokyo (Vaccine strain, Queen Saovabha Memorial Institute, the Thai Red Cross Society) used in this study were grown on Middlebrook 7H11 agar medium or Middlebrook 7H9 liquid medium supplemented with 10\% Middlebrook OADC [Oleic acid Albumin Dextrose Catalase] Enrichment (Difco BBL). Escherichia coli DH5 $\alpha$ [9] and E. coli BL21 ( $\lambda \mathrm{DE} 3$ ) [10], used as a gene cloning host and protein expression host, respectively, were grown in LuriaBertani (LB) medium supplemented with $100 \mu \mathrm{g} / \mathrm{mL}$ of ampicillin or $50 \mu \mathrm{g} / \mathrm{mL}$ of kanamycin where appropriate. Guinea pigs (Dunkin Hartley outbred), female, weight 300 - 400 g were acquired from the National Laboratory Animal Center, Mahidol U., Thailand.

\subsection{PCR Amplification and Cloning of MPT64 (Rv1980c), CFP10 (Rv3874), and ESAT6 (Rv3875) into pET24b}

Genes encoding MPT64 (618 bp, mature peptides Rv1980c), CFP10 (303 bp, Rv3874), and ESAT6 (288 bp, Rv 3875) were PCR amplified from M. tuberculosis H37Rv genomic DNA using three primer pairs (sequences underlined: restriction enzyme recognition sites, sequences in bold: start codon of genes). The primers were designed to clone genes into pET24b to express fusion proteins with $\mathrm{His}_{6}$ at C-termini. For ease of cloning, 5'-primers were designed to include "CATATG" (NdeI recognition site) for which "ATG" is the start codon of Rv3874 and Rv3875. For MPT64 (GenBank: X75361.1), the start codon of mature peptides is "GCG", thus addition of "CATATG" to 5'-primer resulted in an additional methionine at the N-terminus of rMPT64. The 3'primers were designed to exclude the stop codon of the genes with addition of restriction enzyme recognition site (NotI or HindIII).

MPT64-S/5'-primer (5'-AAA CAT ATGGCG CCC AAG ACC TAC TGC GAG GAG-3')

MPT64-ANR/3'-primer (5'-TTT GCG GCC GCG GCC AGC ATC GAG TCG ATC GCG GA-3')

Rv3874Nde5/5'-primer (5'-TAT ACA TAT GGC AGA GAT GAA GAC C-3')

Rv3874Hind3/3'-primer (5'-TAT AAA GCT TGA AGC CCA TTT GCG AG-3')

Rv3875Nde5/5'-primer (5'-TAT ACA TAT GAC AGA GCA GCA GTG G-3')

Rv3875Hind3/3'-primer (5'-TAT AAA GCT TTG CGA ACA TCC CAG T-3')

Fifty microliter reaction mixtures containing $100 \mathrm{ng}$ of DNA template (genomic DNA of M. tuberculosis

H37Rv or M. bovis BCG Tokyo as control), 2.5 units of Takara LA Taq, $0.4 \mathrm{mM}$ of each dNTP, $0.5 \mu \mathrm{M}$ of each 
primer, and $25 \mu \mathrm{L} 2 \times$ GC Buffer II were diluted to $50 \mu \mathrm{L}$ with autoclaved distilled water. The reactions were denatured at $94^{\circ} \mathrm{C}$ for $3 \mathrm{~min}$ and then cycled through 30 rounds of denaturation at $94^{\circ} \mathrm{C}$ for 30 sec, annealing at $60^{\circ} \mathrm{C}$ for $30 \mathrm{sec}$, and extension at $72^{\circ} \mathrm{C}$ for $1 \mathrm{~min}$. These cycles were followed by a final cycle at $72^{\circ} \mathrm{C}$ for $5 \mathrm{~min}$.

The PCR products Rv1980c, Rv3874, and Rv3875 (amplified from genomic DNA of M. tuberculosis H37Rv) were cloned into the PCR cloning vector pGEM-T Easy and were then sub-cloned into the NdeI-NotI or NdeI-HindIII sites of pET24b to generate pET24Rv1980, pET24Rv3874, and pET24Rv3875. Restriction enzyme digestion and DNA sequencing confirmed that the orientation and sequence of the cloned genes were correct.

\subsection{Expression and Purification of Recombinant-His ${ }_{6}$ Proteins}

E. coli BL21 ( $\lambda$ DE3) harboring the recombinant plasmid (pET24Rv1980, pET24Rv3874, or pET24Rv3875) was grown in $50 \mathrm{~mL}$ of LB medium supplemented with $50 \mu \mathrm{g} / \mathrm{mL}$ kanamycin at $37^{\circ} \mathrm{C}$ with shaking (200 rpm) overnight. The next day, the cell cultures were diluted in $500 \mathrm{~mL}$ of fresh medium to an $\mathrm{OD}_{600}$ of 0.05 and incubated at $37^{\circ} \mathrm{C}$ with shaking at $200 \mathrm{rpm}$ until the cultures reached log phase $\left(\mathrm{OD}_{600}\right.$ approx. 0.3 - 0.6). Protein induction was initiated by adding IPTG to a final concentration of $0.5 \mathrm{mM}$ and incubating the cultures at $25^{\circ} \mathrm{C}$ (for pET24Rv1980 and pET24Rv3874) or $37^{\circ} \mathrm{C}$ (for pET24Rv3875) with shaking at 180 rpm for 6 - 12 h. Cells were harvested by centrifugation and washed with PBS.

Cell pellets from $1 \mathrm{~L}$ of cell culture were resuspended with $20 \mathrm{~mL}$ of equilibration buffer (50 mM sodium phosphate buffer, $300 \mathrm{mM} \mathrm{NaCl,} \mathrm{0.1 \%} \mathrm{Triton} \mathrm{X-100,} \mathrm{and} \mathrm{5 \%} \mathrm{glycerol,} \mathrm{pH} \mathrm{7.0).} \mathrm{Lysozyme} \mathrm{and} \mathrm{phenylmethyl-}$ sulfonyl fluoride (PMSF) were added to the cell suspension to a final concentration of $1 \mathrm{mg} / \mathrm{mL}$ and $1 \mathrm{mM}$, respectively. The cell suspensions were incubated on ice for $30 \mathrm{~min}$, and the cells were disrupted by sonication and centrifuged at $4^{\circ} \mathrm{C} / 16000 \times \mathrm{g} / 40 \mathrm{~min}$ to separate the cell debris. The supernatants (clear cell lysate) were then subjected to purification by an Immobilized Metal ion Affinity Column (IMAC) using Talon Superflow Metal Affinity resin (Clontech), as recommended by the manufacturer. Purified protein was analyzed in $12 \%$ or $15 \%$ SDS-PAGE, and the protein bands were visualized by coomassie blue staining.

The elution fractions of purified proteins were pooled and dialyzed against equilibration buffer, pH 7.0 (50 $\mathrm{mM}$ sodium phosphate, $300 \mathrm{mM} \mathrm{NaCl}$ ), until the imidazole concentration was reduced from $150 \mathrm{mM}$ to less than $5 \mathrm{mM}$. The proteins were concentrated, and the buffer was exchanged using a spin column with a molecular weight cutoff (MWCO) of 5000 Da (5 kDa MWCO).

\subsection{Protein Purification by Gel Filtration}

Two proteins, rMPT64 and rESAT6, were further purified by gel filtration using an AKTA purification system with the UNICORN 5.1 program (GE Healthcare). The gel filtration column used was a pre-packed HiLoad 16/60 Superdex 75 pg column, the buffer contained $20 \mathrm{mM}$ sodium phosphate and $150 \mathrm{mM} \mathrm{NaCl}$ at $\mathrm{pH} 7.4$, and the program parameters were as follows: flow rate $=1 \mathrm{~mL} / \mathrm{min}$, injection volume $=1 \mathrm{~mL}$, fraction volume $=3$ $\mathrm{mL}$, and elution volume $=1.2$ column volume.

All purified proteins were filtered by passing them through an Acrodisc Syringe Filter (0.45 $\mu \mathrm{m})$ with low protein binding (Pall). The protein concentration was determined using Lowry’s method. The final protein solution was diluted to $1 \mathrm{mg} / \mathrm{mL}$ before use in animal tests.

\subsection{Cytotoxicity Test}

Proteins purified by IMAC (rMPT64, rCFP10, and rESAT6) and proteins purified by IMAC and gel filtration (rMPT64 and rESAT6) were tested for cytotoxicity at a concentration of $50 \mu \mathrm{g} / \mathrm{mL}$ against Vero cells (African green monkey kidney) at Bioassay Laboratory (BIOTEC, Thailand).

\subsection{Animal Models of TB and Skin Testing of Antigens}

Guinea pigs (Dunkin Hartley outbred, female) were obtained from the Faculty of Veterinary Science, Mahidol University. The in vivo studies were approved by an ethical committee at the Faculty of Veterinary Science, Mahidol University (ethical number MUVS 2009-05), and the experiments were performed in animal BSL3 facilities at the Faculty of Veterinary Science, Mahidol University.

Fifteen guinea pigs weighing 300 - 400 g were divided into 3 groups with 5 animals per group. In groups 1 
and 2, animals were infected (intramuscular injection) on day 1 with $10^{5} \mathrm{CFU}$ of $M$. tuberculosis in $0.1 \mathrm{~mL}$ PBS (pH 7.4) and vaccinated (intradermal injection) with $2 \times 10^{5}$ CFU of M. bovis BCG Tokyo in $0.1 \mathrm{~mL}$ PBS, respectively. Animals in group 3 served as a naïve control. After 4 weeks, both flanks of the animals were shaved, and skin tests were performed by intradermal injection (as shown in Appendix: Figure S1) with either 10 IU PPD (positive control), the specified amounts of the antigens ( 1 or $5 \mu \mathrm{g}$ of single or mixed antigens) diluted in $0.1 \mathrm{~mL}$ PBS, or $0.1 \mathrm{~mL}$ PBS (negative control). The antigens used were IMAC plus gel filtration-purified rMPT64 and rESAT6 and IMAC-purified rCFP10.

Skin test responses (diameter of erythema/redness) were observed and measured after 24, 48, and $72 \mathrm{~h}$. Erythema diameter greater than $0.5 \mathrm{~cm}$ was considered as skin test positive [3]. For ease of interpretation, the erythema formation was determined and scored as follows: $0=$ absent, not perceptible; $1=$ mild redness, diameter $<$ $0.5 \mathrm{~cm} ; 2=$ mild redness, diameter $>0.5 \mathrm{~cm} ; 3=$ redness, diameter $>0.5$ but $<0.8 \mathrm{~cm} ; 4=$ redness, diameter $>$ $0.8 \mathrm{~cm}$; and $5=$ redness, diameter $>0.5 \mathrm{~cm}$, papule. Scores of 0 to 1 were considered "negative", and scores of 2 to 5 were considered a "positive" skin test response.

Skin biopsies were collected at the designated sites (intradermal injection sites) and processed by standard histopathological procedures. The tissue sections were stained with hematoxylin and eosin and observed under a light microscope [11].

\section{Results and Discussion}

\subsection{PCR Amplification of mpt64 (Rv1980c), cfp10 (Rv3874), and esat6 (Rv3875)}

Genes encoding MPT64 (Rv1980c), CFP10 (Rv3874), and ESAT6 (Rv3875) were PCR amplified from M. tuberculosis H37Rv genomic DNA using specific primer pairs as described in Materials and Methods. Control PCR reactions were also performed using genomic DNA of $M$. bovis BCG Tokyo as template. PCR products were analyzed on 1\% agarose gel electrophoresis (Figure 1). The size of PCR fragments of mpt64 (Rv1980c), cfp10 (Rv3874), and esat6 (Rv3875) were 635, 317, and 302 bp, respectively (Figure 1: lane 1, 3, and 5). There was PCR product of $m p b 64$ but not $c f p 10$ and esat 6 from M. bovis BCG Tokyo genomic DNA template (Figure 1 : lane 2, 4, and 6). These results were consistent with the fact that $m p b 64$ is present but $c f p 10$ and esat6 are absent in M. bovis BCG Tokyo [6].

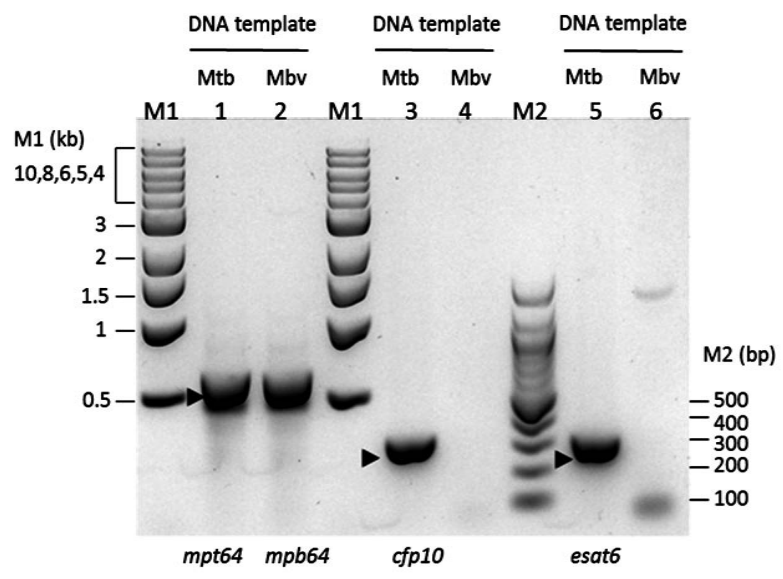

Figure 1. PCR amplification of mpt64 (Rv1980c), cfp10 (Rv3874) and esat6 (Rv3875) from genomic DNA of $M$. tuberculosis H37Rv and $M$. bovis BCG Tokyo. PCR were performed (as described in Materials and Methods) using gene specific primers to amplify mpt64 (Rv1980c), lane 1-2; cfp10 (Rv3874), lane 3-4; and esat6 (Rv3875), lane 5-6. DNA templates used were genomic DNA of $M$. tuberculosis H37Rv (Mtb: lane 1, 3, and 5) and genomic DNA of M. bovis BCG Tokyo (Mbv: lane 2, 4, and 6). Lane M1 and lane M2, $1 \mathrm{~kb}$ DNA Ladder and 100 bp DNA Ladder. Arrows indicate PCR products of mpt64, mpb64, cfp10, and esat6. 


\subsection{Preparation of Recombinant Antigens rMPT64, rCFP10, and rESAT6}

All recombinant antigens (MPT64, CFP10, and ESAT6) were cloned into pET24b and expressed as C-terminal $\mathrm{His}_{6}$-tagged proteins for ease of purification by IMAC. The molecular weights of rMPT64, rCFP10 and rESAT6 were $24.9,12.5$, and $11.5 \mathrm{kDa}$, respectively. All recombinant proteins prepared by IMAC were $>95 \%$ pure as shown in Figure 2. The purification profiles of rMPT64 (Figure 2(a)), rCFP10 (Figure 2(b)), and rESAT6

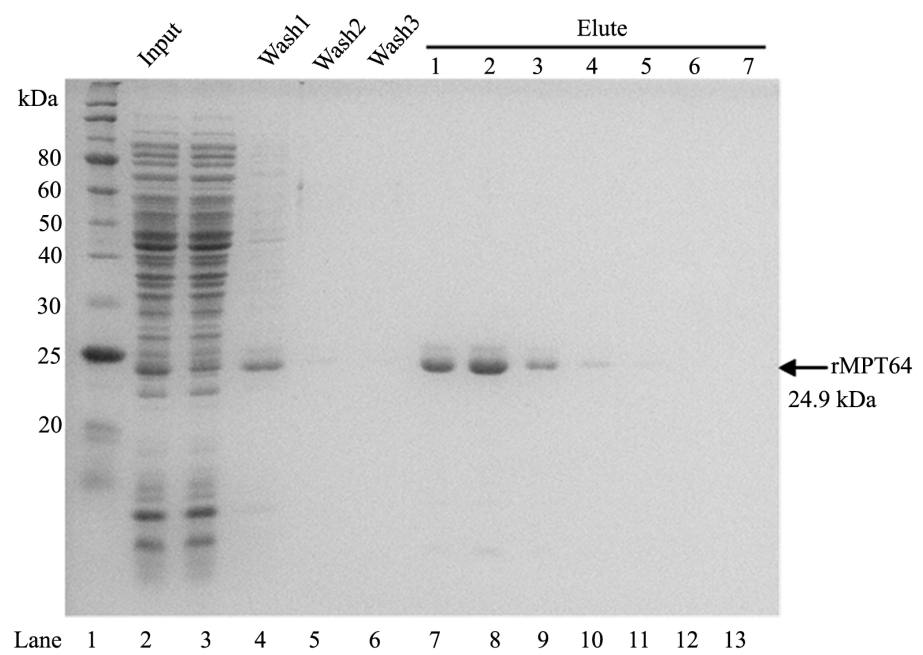

(a)

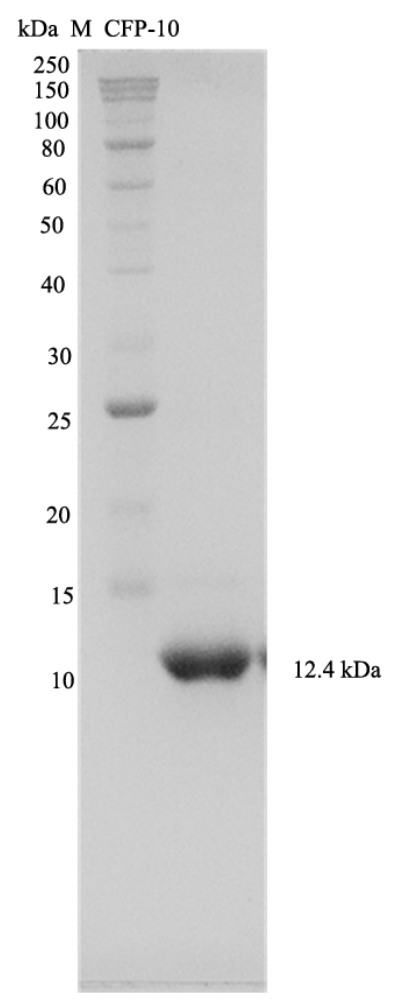

(b)

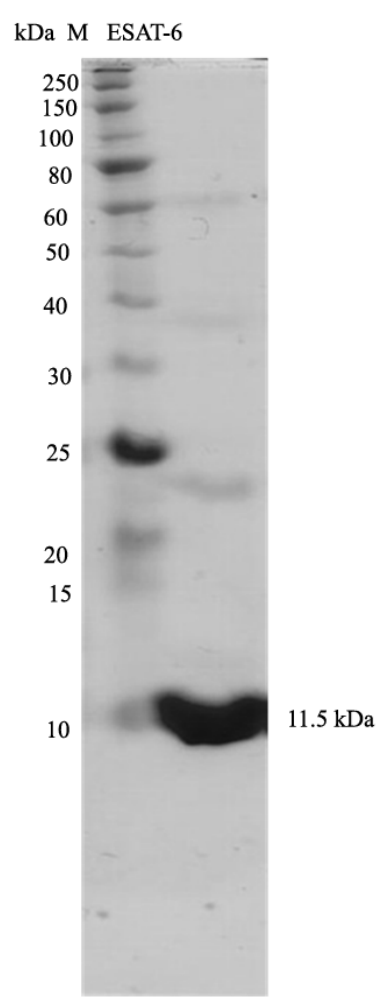

(c)

Figure 2. SDS-PAGE of rMPT64, rCFP10, and rESAT6 purified by IMAC. Proteins were analyzed on 15\% SDS-PAGE and stained with coomassie blue. M, protein MW standard. (a) Purification profile of rMPT64 by IMAC: lane 1, protein MW standard; lane 2, crude protein; lane 3, flow through; lane 4-6, wash; lane 7-13, eluted fraction. The arrow indicates rMPT64 protein; (b) Purified rCFP10; (c) Purified rESAT6. 
(Figure 2(c)) are also provided. For rMPT64 and rESAT6, the proteins were further purified by gel filtration after IMAC purification (data not shown). Proteins purified by IMAC or IMAC plus gel filtration were tested for cytotoxicity against Vero cells, and the results showed that at $50 \mu \mathrm{g} / \mathrm{mL}$, these purified proteins were not toxic (data not shown).

\subsection{Skin Test Responses of Guinea Pig}

Three groups of guinea pigs ( 5 animals per group) were sensitized with PPD or recombinant antigens (rMPT64, rCFP10, and rESAT6) after either 28 days of infection with $M$. tuberculosis H37Rv (group 1), vaccination with M. bovis BCG Tokyo (group 2), or no infection for the naïve control (group 3). Skin test responses were determined and scored after 24, 48, and $72 \mathrm{~h}$ (Appendix: Table S1 and Table S2). In the naïve control group (group 3 ), the animals showed no sign of skin reactions (scoring $=0$, data not shown). All animal groups showed no skin reactions when injected with PBS (scoring $=0$, data not shown). Most of the DTH reaction subsided after 72 h. M. tuberculosis H37Rv-infected animals responded to rMPT64 weaker than they did to PPD. M. bovis BCG Tokyo-vaccinated animals, on the other hand, exhibited a similar DTH response to rMPT64 and to PPD (Figure 3(a) and Figure 3(b)). Our result is in agreement with previous reports [8] [12] that recombinant MPT64 prepared from E. coli elicited a DTH response in guinea pigs infected with $M$. bovis BCG Tokyo. Data from the previous reports also suggested that rMPT64 could replace native MPT64/MPB64 in an in vivo study [8] [12]. Although we did not compare the DTH response obtained from rMPT64-His 6 with native MPT64, our results (Figure 3(b)) indicate that rMPT64-His 6 is active in an in vivo model to the same degree as rMPT64 described in the previous reports.

M. tuberculosis-infected (group 1) animal responses to a single purified antigen were highly variable, and some animals did not respond at all. However, when the three antigens (rMPT64, rCFP10, and rESAT6) were combined, all animals showed positive reactions (Figure 4(a)). On the other hand, one of the M. bovis BCG Tokyo-vaccinated (group 2) animals did not respond to $1 \mu \mathrm{g}$ of combined antigens. However, at higher dose of 5 $\mu \mathrm{g}$ of combined antigens, all animals showed positive DTH response similar to that observed in M. tuberculosis-infected animals (Figure 4(b)). This outcome emphasizes the requirement for having multiple specific antigens to elicit a clear DTH response.

A previous study showed that all $M$. tuberculosis-infected guinea pigs responded well to each of the recombinant antigens, CFP10, ESAT6, and MPT64. When the three antigens were combined, the DTH response was greater than that of any single antigen [5]. However, other studies showed that not all animals infected with $M$. tuberculosis responded to a single antigen [4] [13] [14]. A previous report on the response to rCFP10 was investigated in six M. tuberculosis-infected guinea pigs. During an initial experiment, all six animals responded to rCFP10, but in a second experiment, three animals showed no reactivity to rCFP10 [14]. Another study also showed that in a group of eighteen $M$. tuberculosis-infected guinea pigs, four animals did not respond to rMPT64, and ten animals did not respond to rESAT6, but when these two antigens were combined, strong DTH

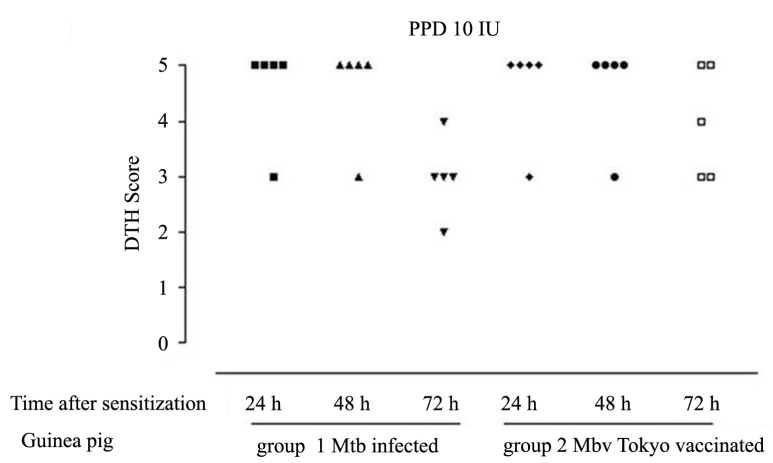

(a)

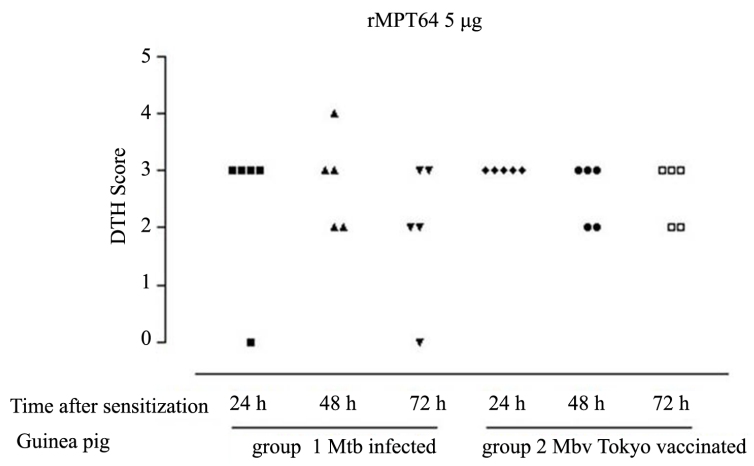

(b)

Figure 3. Analysis of skin test responses of guinea pigs in group 1 (M. tuberculosis infected) and group 2 (M. bovis BCG Tokyo vaccinated). A group of 5 guinea pigs were infected with $M$. tuberculosis or vaccinated with M. bovis BCG Tokyo. After 28 days, the animals were sensitized with antigens, and the skin reactions were recorded after 24,48 , and 72 h. The antigens used were 10 IU PPD (a) and $5 \mu$ g purified rMPT64 (b). 


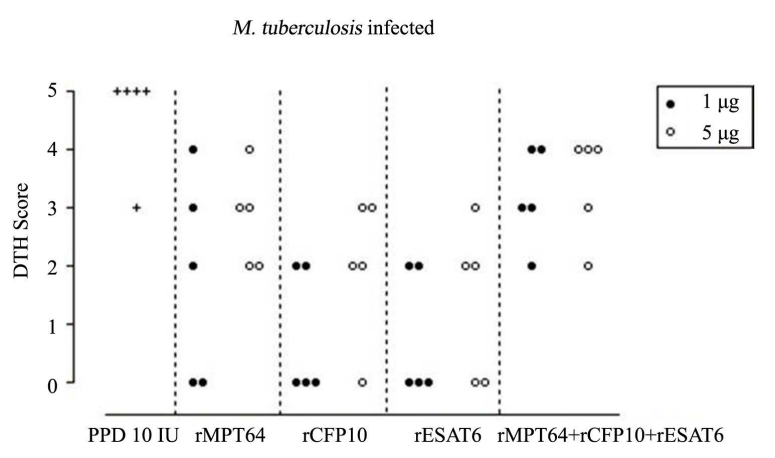

(a)

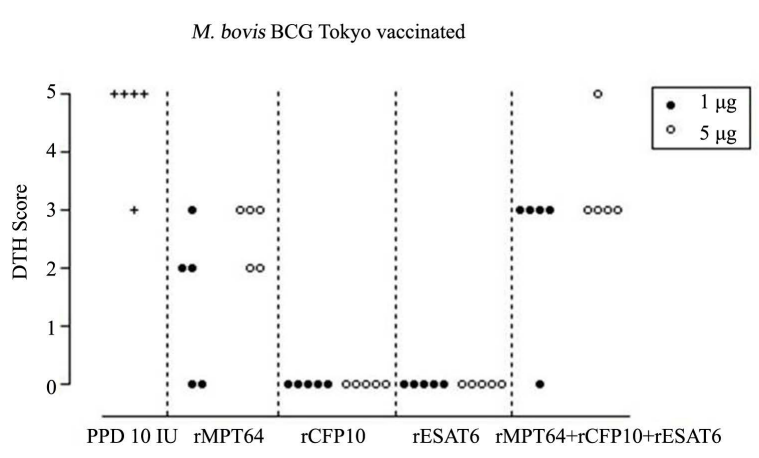

(b)

Figure 4. Analysis of DTH scores of guinea pigs from group 1 (M. tuberculosis infected) and group 2 (M. bovis BCG Tokyo vaccinated) in response to antigens after $48 \mathrm{~h}$. A group of 5 guinea pigs were infected with $M$. tuberculosis (a) or vaccinated with $M$. bovis BCG Tokyo (b). After 28 days, the animals were sensitized with antigens, and the skin reactions were recorded after $48 \mathrm{~h}$. The antigens used were $10 \mathrm{IU}$ PPD, and 1 and $5 \mu \mathrm{g}$ of individual purified antigen (rMPT64, rCFP10, and rESAT6) or a combination of these 3 antigens (mixed of $1 \mu \mathrm{g}$ and mixed of each purified antigen).

reactions were observed in all animals [13]. An investigation of four RD antigens, ESAT6, CFP10, CFP21, and MPT64, showed that these antigens were purified from culture filtrates of M. tuberculosis H37Rv strains and were used to elicit a DTH response in M. tuberculosis H37Rv-infected or M. bovis BCG-vaccinated guinea pigs. As expected, none of these antigens elicited a DTH response in $M$. bovis BCG-vaccinated guinea pigs, as the $\mathrm{RD} 1$ region is absent from the $M$. bovis BCG genome. In addition, not all of the $M$. tuberculosis H37Rv-infected animals responded to individual antigens or a combination of the four antigens (two to three out of four animals responded). However, when two antigens were combined in any possible combination, they could detect all four infected animals, and the highest DTH response was induced by a combination of ESAT6 and CFP10. It was suggested from this study that when combining two or more antigens, their immuno-compatibility (recognition by HLA alleles or competition to antigen-presenting cells) should be taken in consideration [4]. It is possible that variations in the DTH response in guinea pigs may be due to genetic restrictions to some specific antigens and not to others. Another study of six recombinant antigens, MPT63, MPT64, MTC28, MPT32, MPT51, and MPT70, demonstrated the use of these antigens individually or in combination to elicit a DTH response in $M$. bovis BCG Japanese ATCC 35737-immunized guinea pigs. The results showed that the DTH response increased with the number of antigens used [15]. Moreover, a previous study demonstrated that MPT64 alone failed to elicit a DTH response in most human subjects with positive PPD [16].

Our results showed that all animals in group 2 failed to respond to rCFP10 and rESAT6 due to the absence of genes encoding for $c f p 10$ (Rv3874) and esat6 (Rv3875) in M. bovis BCG Tokyo (Figure 4(b)). This result is in agreement with a previous report that investigated the potential use of four antigens, PE35, PPE68, CFP10, and ESAT6, encoded by $M$. tuberculosis-specific RD1 region genes, for active TB diagnosis [17]. In the previous report, the four recombinant antigens were tested for a DTH response in guinea pigs injected with M. tuberculosis, M. bovis BCG, M. avium, and M. vaccae. The results confirmed that these antigens elicited positive DTH responses in the M. tuberculosis-injected group but not in BCG-, M. avium-, and M. vaccae-injected guinea pigs. Thus, RD1 antigens elicited an M. tuberculosis-specific DTH response.

New skin test reagents for TB diagnosis in humans should elicit a strong DTH response similar to that obtained from PPD and also be specific to TB infected patients. The three recombinants antigens, rMPT64, rCFP10, and rESAT6, generated in our study have the potential to be used as new skin test reagents. The rMPT64 induces a DTH response comparable to that elicited by PPD in M. bovis BCG Tokyo-sensitized guinea pigs. The rCFP10 and rESAT6 specifically induce a DTH response in guinea pigs infected with $M$. tuberculosis only. Thus the combined of the three antigens should be further evaluated as skin test reagents in TB diagnosis.

Tissue sections from animals following the intradermal skin test that showed evidence of redness and papule formation were collected at $72 \mathrm{~h}$. Histopathology studies on these lesions showed an infiltration of lymphocytes and macrophages in the dermis, and macrophages were more numerous than lymphocytes. However, the number of infiltrating inflammatory cells was not different between PPD and three other single antigens (rMPT64, rCFP10, and rESAT6) or combined antigens in M. tuberculosis-infected guinea pigs. It was previously reported 
that examination of the skin tissue of BCG-sensitized guinea pigs with DTH in response to rMPB64 found mononuclear cell infiltration similar to that of skin tissue reacting to PPD [7]. The infiltration of mononuclear cells observed in the histopathology of the skin tissue is considered to be classical DTH.

\section{Conclusion}

In this report, we produced purified recombinant proteins rMPT64, rCFP10, and rESAT6 and used them as antigens to stimulate DTH responses in guinea pigs. Our results showed that the purified recombinant proteins were pure and non-toxic to Vero cells and could stimulate a DTH response in animals, confirming previous reports [7] [8] [12]-[15]. It was not surprising that the responses observed from purified proteins were less than those obtained from PPD, because PPD is comprised of $>100$ secreted proteins. Because MPT64 is secreted from actively growing bacteria, our experiment utilized animals that were sensitized with antigens for 4 weeks after vaccination with $M$. bovis BCG Tokyo, during which time the bacteria were still replicating. Thus, positive results were observed because the situation was similar to that in which the animals were infected with $M$. tuberculosis. If skin tests had been performed after 8 weeks, skin tests with rMPT64 would have been negative because the bacteria would have already stopped growing. rCFP10 and rESAT6 are specific antigens secreted from M. tuberculosis, and our results showed that in combination with rMPT64, these antigens increased the DTH response and thus helped to differentiate TB infections. More refined experiments on the amount of single and combined antigens used to stimulate DTH should be performed to formulate a diagnostic test for TB infection. Our study has established the foundation for an improved skin test for TB diagnosis in Thailand.

\section{Competing Interests}

The authors declare that they have no competing interests.

\section{Acknowledgements}

This project was supported by grants from the Cluster Program Management Office (CPMO) and BIOTEC, National Science and Technology Development Agency (NSTDA) (Project P-00-20213 (BT-02-MM-BC-4911) and P-12-01153). The authors thank Duangporn Ungsupravate for technical assistance and thank Dr. Nat Smittipat and Tada Jutayothin for comment and suggestion.

\section{References}

[1] Landi, S. and Held, H.R. (1965) Physicochemical and Biological Studies on Various Preparations of Tuberculin Purified Protein Derivative. Applied Microbiology, 13, 132-139.

[2] Laal, S. and Skeiky, Y.A.W. (2005) Immune-Based Methods. In: Coke, S.T., Eisenach, K.D., McMurray, D.N. and Jacobs Jr., W.R., Eds., Tuberculosis and the Tubercle Bacillus, ASM Press, Washington DC, 71-83. http://dx.doi.org/10.1128/9781555817657.ch5

[3] Coler, R.N., Skeiky, Y.A.W., Ovendale, P.J., Vedvick, T.S., Gervassi, L., Guderian, J., Jen, S., Reed, S.G. and Campos-Neto, A. (2000) Cloning of a Mycobacterium tuberculosis Gene Encoding a Purified Protein Derivative Protein That Elicits Strong Tuberculosis-Specific Delayed-Type Hypersensitivity. Journal of Infectious Diseases, 182, 224233. http://dx.doi.org/10.1086/315677

[4] Kalra, M., Khuller, G.K., Sheikh, J.A. and Verma, I. (2010) Evaluation of Mycobacterium Tuberculosis Specific RD Antigens for Delayed Type Hypersensitivity Responses in Guinea Pig. Indian Journal of Experimental Biology, 48, 117-123.

[5] Stavri, H., Bucurenci, N., Ulea, I., Costache, A., Popa, L. and Popa, M.I. (2012) Use of Recombinant Purified Protein Derivative (PPD) Antigens as Specific Skin Test for Tuberculosis. Indian Journal of Medical Research, 136, 799-807.

[6] Behr, M.A., Wilson, M.A., Gill, W.P., Salamon, H., Schoolnik, G.K., Rane, S. and Small, P.M. (1999) Comparative Genomics of BCG Vaccines by Whole-Genome DNA Microarray. Science, 284, 1520-1523. http://dx.doi.org/10.1126/science.284.5419.1520

[7] Haga, S., Yamaguchi, R., Nagai, S., Matsuo, K., Yamazaki, A. and Nakamura, R.M. (1995) Delayed-Type Hypersensitivity to a Recombinant Mycobacterial Antigen, MPB64, in Guinea Pigs Sensitized to Mycobacterium tuberculosis or Mycobacterium bovis BCG. Journal of Leukocyte Biology, 57, 221-225.

[8] Geisbrecht, B.V., Nikonenko, B., Samala, R., Nakamura, R., Nacy, C.A. and Sacksteder, K.A. (2006) Design and Optimization of a Recombinant System for Large-Scale Production of the MPT64 Antigen from Mycobacterium tubercu- 
losis. Protein Expression and Purification, 46, 64-72. http://dx.doi.org/10.1016/j.pep.2005.08.011

[9] Woodcock, D.M., Crowther, P.J., Doherty, J., Jefferson, S., DeCruz, E., Noyer-Weidner, M., Smith, S.S., Michael, M.Z. and Graham, M.W. (1989) Quantitative Evaluation of Escherichia coli Host Strains for Tolerance to Cytosine Methylation in Plasmid and Phage Recombinants. Nucleic Acids Research, 17, 3469-3478.

http://dx.doi.org/10.1093/nar/17.9.3469

[10] Studier, F.W., Rosenberg, A.H., Dunn, J.J. and Bubendorff, J.W. (1990) Use of T7 RNA Polymerase to Direct Expression of Cloned Genes. Methods in Enzymology, 185, 60-89. http://dx.doi.org/10.1016/0076-6879(90)85008-C

[11] Fischer, A.H., Jacobson, K.A., Rose, J. and Zeller, R. (2008) Hematoxylin and Eosin Staining of Tissue and Cell Sections. CSH Protocols.

[12] Oettinger, T., Holm, A., Mtoni, I.M., Andersen, A.B. and Hasløov, K. (1995) Mapping of the Delayed-Type Hypersensitivity-Inducing Epitope of Secreted Protein MPT64 from Mycobacterium tuberculosis. Infection and Immunity, 63, 4613-4618.

[13] Elhay, M.J., Oettinger, T. and Andersen, P. (1998) Delayed-Type Hypersensitivity Responses to ESAT-6 and MPT64 from Mycobacterium tuberculosis in the Guinea Pig. Infection and Immunity, 66, 3454-3456.

[14] Colangeli, R., Spencer, J.S., Bifani, P., Williams, A., Lyashchenko Keen, M.A., Hill, P.J., Belisle, J. and Gennaro, M.L. (2000) MTSA-10, the Product of the Rv3874 Gene of Mycobacterium tuberculosis, Elicits Tuberculosis-Specific, Delayed-Type Hypersensitivity in Guinea Pigs. Infection and Immunity, 68, 990-993. http://dx.doi.org/10.1128/IAI.68.2.990-993.2000

[15] Lyashchenko, K., Manca, C., Colangeli, R., Heijbel, A., Williams, A. and Gennaro, M.L. (1998) Use of Mycobacterium tuberculosis Complex-Specific Antigens for a Skin Test Specific for Tuberculosis. Infection and Immunity, 66, 3606-3610.

[16] Wilcke, J.T., Jensen, B.N., Ravn, P., Andersen, A.B. and Hasløv, K. (1996) Clinical Evaluation of MPT-64 and MPT59, Two Proteins Secreted from Mycobacterium tuberculosis, for Skin Test Reagents. Tubercle and Lung Disease, 77, 250-256. http://dx.doi.org/10.1016/S0962-8479(96)90009-X

[17] Hanif, S.N., Al-Attiyah, R. and Mustafa, A.S. (2010) Species-Specific Antigenic Mycobacterium tuberculosis Proteins Tested by Delayed-Type Hypersensitivity Response. International Journal of Tuberculosis and Lung Disease, 14, 489494.

\section{Abbreviations}

PPD: Purified Protein Derivative

MPT64: immunogenic protein from M. tuberculosis identical to MPB64 from M. bovis

ESAT6: Early Secreted Antigen Target 6

CFP10: Culture Filtrate Protein 10

PBS: Phosphate Buffer Saline

CFU: Colony Forming Unit

BCG: Bacille Calmette-Guérin

IMAC: Immobilized Metal ion Affinity Chromatography

PCR: Polymerase Chain Reaction

DTH: Delayed-Type Hypersensitivity

RD: Region of Difference 


\section{Appendix}

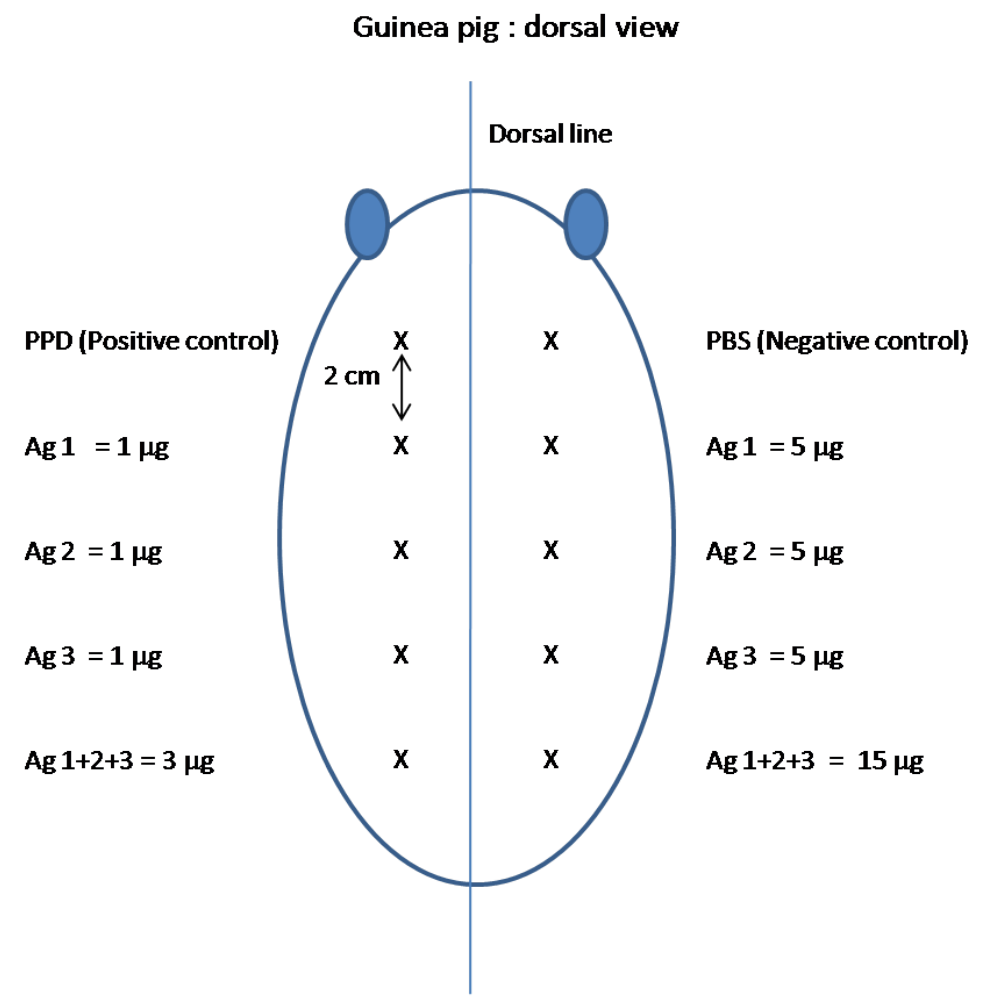

$\mathrm{X}$ = intradermal injection spot; each spot is $\mathbf{2} \mathbf{~ c m}$ apart

Figure S1. Intradermal injection of antigens on both flanks of a guinea pig. PPD and PBS were used as the positive and negative controls, respectively. Purified antigens were tested individually or in combination. The antigen concentrations used were 1 and $5 \mu \mathrm{g}$. Ag1, rMPT64; Ag2, rCFP10; Ag3, rESAT6. 
W. Yindeeyoungyeon et al.

Table S1. Guinea pig group 1 (M. tuberculosis H37Rv infected): scores of the skin test responses after the guinea pigs were sensitized with antigens for 24,48 , and $72 \mathrm{~h}$.

\begin{tabular}{|c|c|c|c|c|c|c|c|}
\hline \multirow{2}{*}{ Antigen } & \multirow{2}{*}{ Amount of Ag } & \multirow{2}{*}{$\begin{array}{l}\text { Time after } \\
\text { sensitization }\end{array}$} & \multicolumn{5}{|c|}{ Skin test response score } \\
\hline & & & Guinea pig \#1 & Guinea pig \#2 & Guinea pig \#3 & Guinea pig \#4 & Guinea pig \#5 \\
\hline \multirow[t]{3}{*}{ PPD } & $10 \mathrm{IU}$ & $24 \mathrm{~h}$ & 5 & 5 & 5 & 5 & 3 \\
\hline & & $48 \mathrm{~h}$ & 5 & 5 & 5 & 3 & 5 \\
\hline & & $72 \mathrm{~h}$ & 3 & 3 & 4 & 2 & 3 \\
\hline \multirow[t]{6}{*}{ rMPT64 } & $1 \mu \mathrm{g}$ & $24 \mathrm{~h}$ & 3 & 3 & 0 & 3 & 4 \\
\hline & & $48 \mathrm{~h}$ & 0 & 4 & 0 & 2 & 3 \\
\hline & & $72 \mathrm{~h}$ & 0 & 2 & 0 & 0 & 2 \\
\hline & $5 \mu \mathrm{g}$ & $24 \mathrm{~h}$ & 3 & 3 & 0 & 3 & 3 \\
\hline & & $48 \mathrm{~h}$ & 2 & 3 & 2 & 3 & 4 \\
\hline & & $72 \mathrm{~h}$ & 0 & 3 & 2 & 2 & 3 \\
\hline \multirow[t]{6}{*}{ rCFP10 } & $1 \mu \mathrm{g}$ & $24 \mathrm{~h}$ & 0 & 0 & 2 & 0 & 2 \\
\hline & & $48 \mathrm{~h}$ & 0 & 0 & 2 & 0 & 2 \\
\hline & & $72 \mathrm{~h}$ & 0 & 0 & 0 & 0 & 0 \\
\hline & $5 \mu \mathrm{g}$ & $24 \mathrm{~h}$ & 3 & 3 & 2 & 3 & 0 \\
\hline & & $48 \mathrm{~h}$ & 2 & 3 & 2 & 3 & 0 \\
\hline & & $72 \mathrm{~h}$ & 0 & 0 & 0 & 0 & 0 \\
\hline \multirow[t]{6}{*}{ rESAT6 } & $1 \mu \mathrm{g}$ & $24 \mathrm{~h}$ & 0 & 2 & 2 & 0 & 3 \\
\hline & & $48 \mathrm{~h}$ & 0 & 0 & 2 & 0 & 2 \\
\hline & & $72 \mathrm{~h}$ & 0 & 0 & 0 & 0 & 0 \\
\hline & $5 \mu \mathrm{g}$ & $24 \mathrm{~h}$ & 0 & 2 & 3 & 0 & 0 \\
\hline & & $48 \mathrm{~h}$ & 0 & 2 & 3 & 0 & 2 \\
\hline & & $72 \mathrm{~h}$ & 0 & 2 & 0 & 0 & 0 \\
\hline rMPT64+ & $1+1+1 \mu \mathrm{g}$ & $24 \mathrm{~h}$ & 3 & 3 & 3 & 3 & 4 \\
\hline rCFP10+ & & $48 \mathrm{~h}$ & 3 & 4 & 3 & 2 & 4 \\
\hline \multirow[t]{4}{*}{ rESAT6 } & & $72 \mathrm{~h}$ & 0 & 2 & 3 & 0 & 3 \\
\hline & $5+5+5 \mu \mathrm{g}$ & $24 \mathrm{~h}$ & 3 & 3 & 3 & 4 & 3 \\
\hline & & $48 \mathrm{~h}$ & 2 & 4 & 4 & 4 & 3 \\
\hline & & $72 \mathrm{~h}$ & 0 & 4 & 4 & 3 & 2 \\
\hline
\end{tabular}


W. Yindeeyoungyeon et al.

Table S2. Guinea pig group 2 (M. bovis BCG Tokyo vaccinated): scores of the skin test responses after the guinea pigs were sensitized with antigens for 24,48 , and $72 \mathrm{~h}$.

\begin{tabular}{|c|c|c|c|c|c|c|c|}
\hline \multirow{2}{*}{ Antigen } & \multirow{2}{*}{ Amount of Ag } & \multirow{2}{*}{$\begin{array}{l}\text { Time after } \\
\text { sensitization }\end{array}$} & \multicolumn{5}{|c|}{ Skin test response score } \\
\hline & & & Guinea pig \#1 & Guinea pig \#2 & Guinea pig \#3 & Guinea pig \#4 & Guinea pig \#5 \\
\hline \multirow[t]{3}{*}{ PPD } & $10 \mathrm{IU}$ & $24 \mathrm{~h}$ & 5 & 3 & 5 & 5 & 5 \\
\hline & & $48 \mathrm{~h}$ & 5 & 5 & 5 & 3 & 5 \\
\hline & & $72 \mathrm{~h}$ & 4 & 3 & 5 & 3 & 5 \\
\hline \multirow[t]{6}{*}{ rMPT64 } & $1 \mu \mathrm{g}$ & $24 \mathrm{~h}$ & 3 & 3 & 3 & 3 & 3 \\
\hline & & $48 \mathrm{~h}$ & 3 & 2 & 2 & 0 & 0 \\
\hline & & $72 \mathrm{~h}$ & 4 & 2 & 2 & 0 & 0 \\
\hline & $5 \mu \mathrm{g}$ & $24 \mathrm{~h}$ & 3 & 3 & 3 & 3 & 3 \\
\hline & & $48 \mathrm{~h}$ & 3 & 2 & 2 & 3 & 3 \\
\hline & & $72 \mathrm{~h}$ & 3 & 2 & 2 & 3 & 3 \\
\hline \multirow[t]{6}{*}{ rCFP10 } & $1 \mu \mathrm{g}$ & $24 \mathrm{~h}$ & 0 & 0 & 0 & 0 & 0 \\
\hline & & $48 \mathrm{~h}$ & 0 & 0 & 0 & 0 & 0 \\
\hline & & $72 \mathrm{~h}$ & 0 & 0 & 0 & 0 & 0 \\
\hline & $5 \mu \mathrm{g}$ & $24 \mathrm{~h}$ & 0 & 0 & 0 & 0 & 0 \\
\hline & & $48 \mathrm{~h}$ & 0 & 0 & 0 & 0 & 0 \\
\hline & & $72 \mathrm{~h}$ & 0 & 0 & 0 & 0 & 0 \\
\hline \multirow[t]{6}{*}{ rESAT6 } & $1 \mu \mathrm{g}$ & $24 \mathrm{~h}$ & 0 & 0 & 0 & 0 & 0 \\
\hline & & $48 \mathrm{~h}$ & 0 & 0 & 0 & 0 & 0 \\
\hline & & $72 \mathrm{~h}$ & 0 & 0 & 0 & 0 & 0 \\
\hline & $5 \mu \mathrm{g}$ & $24 \mathrm{~h}$ & 0 & 0 & 0 & 0 & 0 \\
\hline & & $48 \mathrm{~h}$ & 0 & 0 & 0 & 0 & 0 \\
\hline & & $72 \mathrm{~h}$ & 0 & 0 & 0 & 0 & 0 \\
\hline rMPT64+ & $1+1+1 \mu \mathrm{g}$ & $24 \mathrm{~h}$ & 3 & 3 & 0 & 3 & 3 \\
\hline rCFP10+ & & $48 \mathrm{~h}$ & 3 & 3 & 0 & 3 & 3 \\
\hline \multirow[t]{4}{*}{ rESAT6 } & & $72 \mathrm{~h}$ & 3 & 3 & 0 & 3 & 3 \\
\hline & $5+5+5 \mu \mathrm{g}$ & $24 \mathrm{~h}$ & 3 & 3 & 3 & 3 & 3 \\
\hline & & $48 \mathrm{~h}$ & 3 & 5 & 3 & 3 & 3 \\
\hline & & $72 \mathrm{~h}$ & 3 & 3 & 3 & 2 & 2 \\
\hline
\end{tabular}

